\title{
Interferon Regulatory Factor 5 and Renin-Angiotensin-Aldosterone System Polymorphisms in Coronary Artery Disease: An Overview of Experimental and Clinical Studies
}

\author{
Jorge Luis Bermudez-Gonzalez ${ }^{1,2}$, Rodrigo Dagio-Cuellar2, Cristina Villarreal-Guerrero², \\ Ana Gilabert-Garcia ${ }^{2}$, Luis Angel Ferral-Barbabosa ${ }^{2}$, Joaquin Berarducci ${ }^{1}$, Jose Luis Siller-Nava ${ }^{1}$, \\ Jose Antonio Luna-Alvarez-Amezquita1, Javier Iván Armenta-Moreno', Nilda Espínola-Zavaleta1, \\ Erick Alexanderson-Rosas ${ }^{1,2}$, Juan Ignacio Straface ${ }^{1 *}$
}

${ }^{1}$ National Institute of Cardiology Ignacio Chavez, Mexico City, Mexico

${ }^{2}$ Medicine Faculty of National Autonomous University of Mexico, Mexico City, Mexico

Email: *dr.straface@outlook.com

How to cite this paper: Bermudez-Gonzalez, J.L., Dagio-Cuellar, R., Villarreal-Guerrero, C., Gilabert-Garcia, A., Ferral-Barbabosa, L.A., Berarducci, J., Siller-Nava, J.L., Luna-Alvarez-Amezquita, J.A., Armenta-Moreno, J.I., Espínola-Zavaleta, N., Alexanderson-Rosas, E. and Straface, J.I. (2021) Interferon Regulatory Factor 5 and ReninAngiotensin-Aldosterone System Polymorphisms in Coronary Artery Disease: An Overview of Experimental and Clinical Studies. World Journal of Cardiovascular Diseases, 11, 332-341.

https://doi.org/10.4236/wicd.2021.117032

Received: June 7, 2021

Accepted: July 6, 2021

Published: July 9, 2021

Copyright $\odot 2021$ by author(s) and Scientific Research Publishing Inc. This work is licensed under the Creative Commons Attribution International License (CC BY 4.0).

http://creativecommons.org/licenses/by/4.0/

\begin{abstract}
Heart diseases are the main cause of mortality in Mexico, being coronary heart disease the most frequent in the country. Its high prevalence makes important the study of the pathophysiology and the search for prognostic factors. Different genes and polymorphisms promote atherogenesis and coronary artery disease, they affect inflammatory and vascular pathological processes. Interferon regulatory factor 5 (IRF5) is associated with coronary heart disease, it promotes chronic inflammation and cytokines release; it could trigger immune reactions and its activating receptors express in the vascular endothelium. Besides, polymorphisms in the renin-angiotensin-aldosterone system (RAAS) are implied with coronary disease, they are found in angiotensinogen (AGT), angiotensin II type 1 receptor (AT1R), angiotensin II type 2 receptor (AT2R), and angiotensin-converting enzyme (ACE) genes. These genetic polymorphisms are associated with a prothrombotic state, endothelial dysfunction, and immune activation. Multiple experimental studies showed that chronic activation of RAAS and chronic expression of IRF5 generates an environment prone to the development of atherosclerosis, and autoimmune and cardiovascular diseases. Studying these specific genes and their relationship with coronary heart disease will allow a better understanding of the pathological process and possibly the quest for new treatments.
\end{abstract}

\section{Keywords}

Interferon Regulatory Factor 5 (IRF5), Angiotensin-Converting Enzyme 
(ACE), Angiotensinogen (AGT), Angiotensin II Type 1 Receptor (AT1R), Angiotensin II Type 2 Receptor (AT2R), Polymorphisms

\section{Introduction}

Globally, coronary heart disease represents $56 \%$ of the reported deaths according to the World Health Organization (WHO), ischemic heart disease and stroke being the principal causes. On the latest Mexican National Health Survey, the mean LDL levels have been increasing at a younger age than in previous years [1]. Systemic arterial hypertension prevalence is increasing in our population, accounting for $25.5 \%$ of the population. These diseases constitute well-known risk factors for the development of ischemic cardiopathy and coronary heart disease [1]. In Mexico, cardiopathies are the first cause of mortality, produce the $15.7 \%$ of all deaths [2]. The high mortality rate associated with coronary heart disease is an important public health issue in Mexico. The lack of early preventive attention given to the disease takes a leading role on the high morbidity and mortality rates associated to coronary heart disease [2]. Proper identification and research of the associated polymorphisms result of prime relevance for the development and severity of the disease.

Interferon Regulatory Factor 5 (IRF5) has been studied in the past due to the great number of polymorphisms presented and its association with multiple autoimmune and inflammatory diseases [3]. Recently, this regulatory factor has gained importance in studying coronary heart disease owing to its influence in the pathophysiology of chronic pro-inflammatory reactions. Its increased expression is related to the activation of receptors on the vascular endothelium, furthermore, initiating immunity processes also present at the pathophysiology of the formation of atherosclerotic plaques at the coronary arteries.

On the other hand, different polymorphisms of multiple genes in the reninangiotensin-aldosterone system (RAAS) have been associated with a greater incidence of coronary heart disease, thanks to its direct and indirect influence on causing a prothrombotic state, endothelial disfunction and immune system activation. The main polymorphisms associated with the vascular pathological pathways belong to Angiotensinogen (AGT), Angiotensin II Type 1 receptor (AT1R), Angiotensin II Type 2 receptor (AT2R), and the Angiotensin-Converting Enzyme (ACE) [4].

In the present discussion we analyze the most relevant results of experimental and clinical studies related to the previously mentioned polymorphisms. Based on this knowledge, future research could aim to identify new clinical scenarios related to specific polymorphisms in these genes. This "genetic-centered" approach could establish a new way for understanding pathophysiological grounds of the disease and develop newer considerations for predicting factors of disease and achieving preventing and curative therapies in the future. 


\section{Interferon Regulatory Factor $\mathbf{5}$}

IRF5 is part of a big family of interferon transcription factors which were initially associated with antiviral responses; induced mainly by IFN production. It is found in eleven different isoforms, which utilize various promoters, express in different proportions among different cells, and give rise to different functions or responses [3]. Moreover, IRF5 is usually activated by the pattern recognition receptors (PRRs) that recognize molecules from pathogenic microorganisms and cellular damage molecules. Common examples of these receptors are toll-like receptors (TLR), particularly TLR4 that recognizes bacterial LPS; and the NODlike receptors, which detect peptidoglycan-derived molecules, cytosolic ATP, intracellular crystals, and ionic concentration changes [5]. Its expression is also induced by the granulocyte-macrophage colony stimulating factor (GM-CSF) and interferon-gamma. IRF5 protein is activated by MyD88 molecule in response to PRRs stimulation, moving to the nucleus where it mediates the genetical expression needed for more specialized responses. In this way, IRF5 promotes the production of neutrophile recruiting chemokines, and the expression of a proinflammatory phenotype on macrophages (M1), secondarily reducing the homeostatic repairing activity (M2). The expression of IRF5 promotes, broadly speaking, the secretion of IFN-1, TNF- $\gamma$, IL-6, IL-12, and IL-23 (proinflammatory chemokines), simultaneously represses the secretion of IL-10 and TGF- $B$ (anti-inflammatory chemokines). IRF5 is associated to a maintained production of TNF- $\gamma$, and the polarization to Th1 and Th17 lymphocytic responses [6]. There are at this moment 9 members of this family described in the literature, all of them having in common conserved multidomains necessary for recognizing DNA, mediating protein interactions and forming protein complexes with transcriptional functions. Now, IRF5 expression may be useful as an inflammatory marker due to the increased classical macrophage response it provokes. The physiologic implications attributed to IRF5 have been studied in animal models as well as in human beings, getting to know distinctive characteristics associated with diverse pathologies.

According to studies conducted on mice with atherosclerosis and IRF5-deletion $(-/-)$, the lack of expression of IRF5 is ligated with a minor incidence of vascular lesions and smaller size of necrotic regions. Mainly, this happens because of the alternative expression of macrophages (M2) that promotes epherocytosis (i.e., apoptotic cell elimination), and removal of necrotic tissues or cellular detritus by phagocytes [7]. In further studies with two murine models with atherosclerosis, the murine IRF5 gene was observed to impair the efficacy of phagocytic action and promote atherosclerotic lesions. The anterior is caused by the role of IRF5 on promoting a proinflammatory state mediated by $\mathrm{CD} 11 \mathrm{c}+$ macrophages and propitiating necrotic nucleus expansion of the lesions [7].

It has been also demonstrated that IRF5 plays a leading role on antigen-induced arthritis (AIA) and in pulmonary lesions provoked by LPS. On mice with these diseases, those which lacked IRF5 had a reduced flow of neutrophils in the 
acute phase [9]. Inflamed tissues had a lesser neutrophile count presumably because of the reduced number of chemokines in charge of neutrophile recruitment, for example, chemokine ligand 1 (CXC). A similar reduction was observed in neutrophile recruitment on the serum transference induced arthritis model $\mathrm{K} / \mathrm{BxN}$; in which mice who lacked IRF5 had a reduction in severity of the arthritis flares [10]. This is explained because the mice without IRF5 expression exhibit reduced Th1, Th17 and $\gamma \delta$ lymphocytic responses on the chronic phases of AIA [8]. Also, IRF5 positive expression was determined to be a fundamental factor for MHCII + phenotype establishment on dormant state macrophages of the knee joint, as well as in the monocyte-derived macrophages during antigen-induced arthritis episodes. These studies strengthen evidence in favor of direct IRF5 inhibition as a treatment for chronic inflammation induced by macrophages. There is also a suggestion that more acute presentations of inflammation, included pulmonary lesion, might also improve with IRF5 directed inhibition [9].

On human studies, IRF5 single nucleotide polymorphisms are associated with an enormous variety of autoimmune diseases, included systemic erythematosus lupus, rheumatoid arthritis, and intestinal inflammatory disease, to mention some of them [11]. It has been reported that IRF5 is one of the most significative expression quantitative trait loci (eQTL) found on human myeloid cells stimulated with LPS [12] [13]. The anterior contributes to the variability in immune responses, emphasizing its key regulator role in inflammatory responses induced by TLR. This way, IRF5 constitutes a potential pharmacological target for disease modifying drugs in the treatment of autoimmune diseases, offering better specificity and selectivity over a wide spectrum targets [8].

Another study analyzed samples of visceral adipose tissue from 56 patients with different weight ranges [14]. IRF5 gene and IRF5 protein expression was quantified, and a clear increase in the expression of IRF5 was found on patients with high baseline body fat and increased body mass index (BMI), indicative of overweight and obesity. Expression of other inflammatory markers were also analyzed, such as TNF $\alpha$, IL-6, IL-I $\beta$, IL-18, CXCL8, CCL2/5, and C-reactive protein. The anterior molecules proportionally corelated with the expression of IRF5 (gene and protein). On the other hand, adiponectin expression had an inversely proportional relation with IRF5 expression. Four of the patients in the study had a previous history of hypertension and another three presented with dyslipidemia. These findings demonstrate that people who are overweight or obese possess an inflammatory metabolic state within fat tissue. However, further analysis will be needed to demonstrate similar expression of inflammatory markers at a systemic level. Unfortunately, patients with history of cardiac disease were excluded from the study. Similar conclusions have been found on multiple studies, where IRF5 expression on macrophages has a pathological outcome on diet-induced obesity [15], despite having a significant protective action against inflammation in the airways [16]. 
Before the above-cited study, data of IRF5 influence on cardiovascular diseases had already been collected. IRF5 levels were compared between atherosclerotic tissue and a control group. Simultaneously, IRF5 protein expression was measured in relation to IRF5 genotype. The mean IRF5 mRNA expression was significatively greater on the carotid atherosclerotic tissue compared to the control group. Moreover, there was no difference found of IRF5 expression between men and women [17].

The anterior findings are better understood and justified considering that atherosclerotic disease is a complex and multifactorial condition. Multiple noxious stimuli conduct to the development of coronary heart disease (Figure 1), such as excessive inflammatory response and fibroproliferative reaction in the arterial subintimal space, promoting thrombus formation. After an acute myocardial ischemic event, cellular debris activate local immune cells through TLRs, being TLR2 and 4 the most frequent in the heart. Both receptors-specially TLR4start the activation signaling cascade of IRF5, leading to the proinflammatory processes previously commented [18].

\section{Renin-Angiotensin-Aldosterone System}

The renin-angiotensin-aldosterone system (RAAS) has a fundamental homeostatic role on the human body, it oversees the long-term regulation of arterial blood pressure, sodium concentration and extracellular fluid volume. Angiotensinogen is the protein that initiates the activation cascade, it is produced in the liver, and is released under low blood pressure states, by action of the sympathetic nervous system, or by changes of the interstitial sodium concentration. Renin, released by the kidneys under the previously stated stimuli, regulates the transformation of angiotensinogen to angiotensin I. Further in the biochemical pathway, angiotensin-converting enzyme (ACE) converse angiotensin I to angiotensin II, the most active form. The ACE is mainly produced by the pulmonary endothelium,

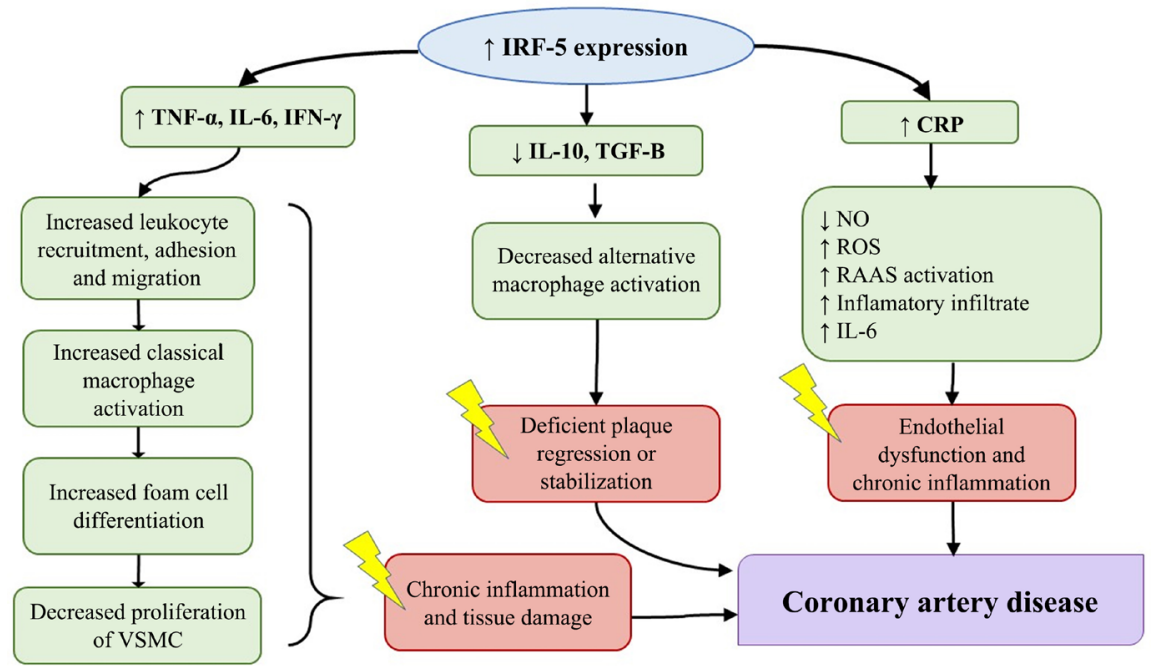

Figure 1. Effects of IRF-5 expression. C reactive protein (CRP); vascular smooth muscle cells (VSMC); reactive oxygen species (ROS); nitric oxide (NO). 
but it is also expressed in the kidneys, the adrenal glands, the brain, and the heart. ACE also diminishes the synthesis of nitric oxide by degrading bradykinin, and consequently produces local vasoconstriction (Figure 2).

Angiotensin II generates broad effects that depend on the stimulated receptor. AT1R is found in the kidneys, heart, vascular smooth muscle, brain, adrenal glands, platelets, adipose tissue, and the placenta. It promotes vasoconstriction, sodium and water retention (via aldosterone-release induction), reactive oxygen species formation (ROS), fibrosis, inflammation, cellular growth and proliferation, suppression of renin-release as a regulatory mechanism, etc. Meanwhile, AT2R expresses in uterus, adrenal glands, CNS, heart, and kidneys. Its activation produces vasodilation, damaged tissue repair, inhibition of cellular proliferation, and stimulates kidney and urinary tract development. There are other angiotensin II receptors, which have a minor expression in the human body. An example with clinical significance is the angiotensin II type 4 receptor (AT4R), which reduces fibrinolysis effectivity [19].

A study evaluated the relationship between certain RAAS gene expression polymorphisms, C-reactive protein (CRP) serum levels, and coronary heart disease severity [20]. CRP is secreted by atherosclerotic plaques, activates circulating monocytes, promotes endothelial dysfunction, and provokes cellular matrix remodeling. It was observed a proportional relationship between CRP serum levels and the number of coronary arteries affected. The same study found an association

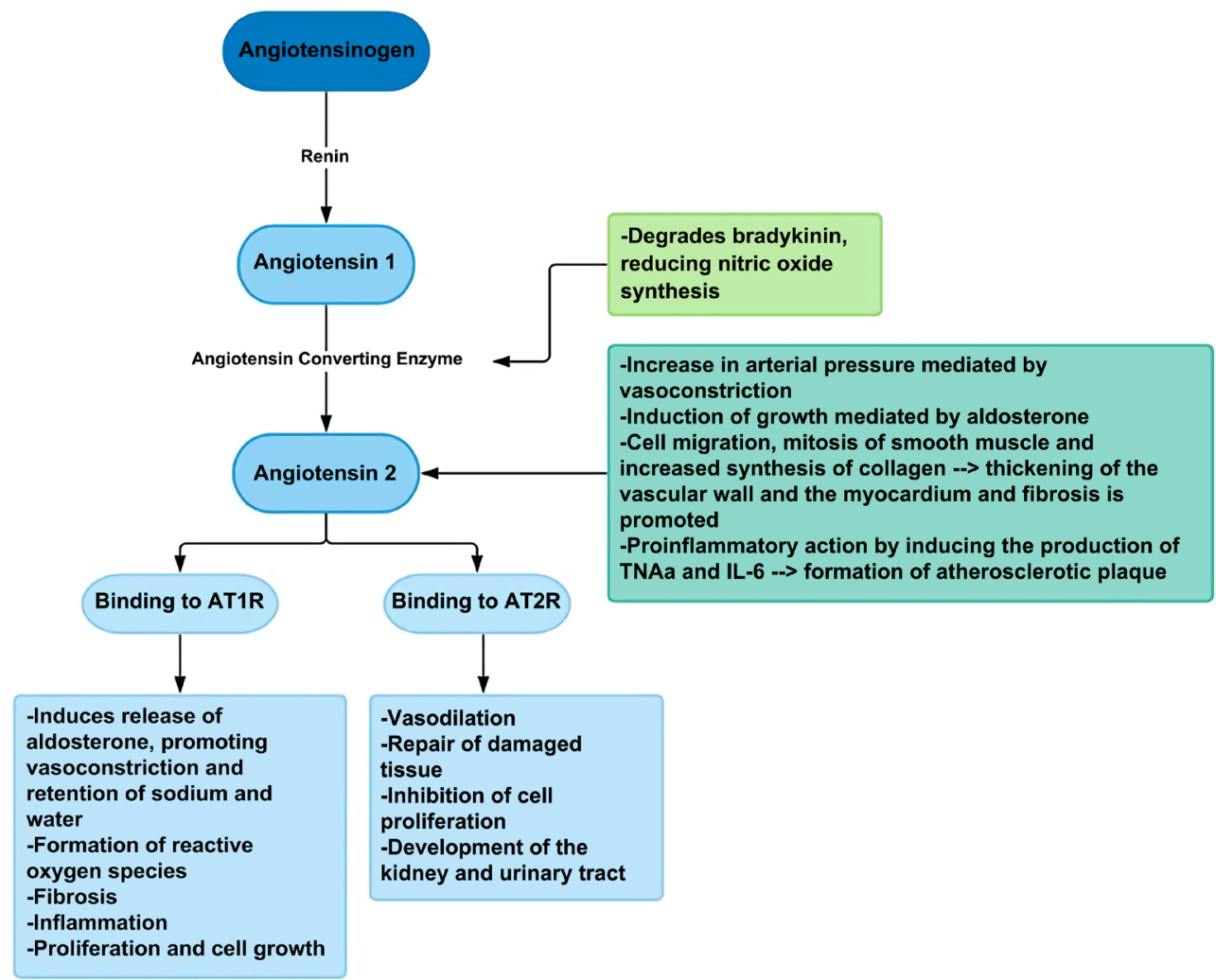

Figure 2. Effects of renin-angiotensin-aldosterone system. Angiotensin type 1 receptor (AT1R), angiotensin type 2 receptor (AT2R). 
between the AGT M235T allele expression and an increased susceptibility to present coronary heart disease by AT1R activation, as well as increased ROS and CRP release. The AGT M235T polymorphism was associated with an increased risk of coronary heart disease in Brazilian Caucasic [21] and Egyptian population [22].

Another study found a correlation between ACE, APO E gene polymorphisms (E2, E3, E4 and g.-219G/T), and myocardial perfusion. 410 patients were studied with single-photon emission computed tomography (SPECT) and myocardial perfusion was evaluated at rest and exercise settings, calculating the summed stress score (SSS), the summed rest score (SRS), and the summed differential score (SDS). The homozygous carriers for ACE D allele had increased mean values of SSS $(\mathrm{P}<0.001)$ and SDS $(\mathrm{P}<0.001)$. After adjusting for clinical and demographical data, and using multiple lineal regression analysis, authors concluded that both ACE D/D and APO E were independent predictors with cumulative contribution for SSS and SDS estimation. Also, logistic regression analysis revealed that the 3 genotypes had an independent predictive capacity of abnormal SSS (SSS $\geq 2$ ). Moreover, a clear majority of patients with moderate to severe SPECT studies (SSSS $\geq 9$ ) were homozygous for the D/D allele, while the I/I and I/D distribution was similar. D/D allele was recognized to be associated with a high plasma ACE activity, an increased blood pressure, and increased risk of plaque rupture on multiple populations. The study concluded that ACE D/D genotype can modify myocardial perfusion capacity promoting an increase in cardiovascular events. These data account for the first evidence of an association and significative cumulative contribution between the previously mentioned genotypes and anomalies of myocardial perfusion. Finally, E4 allele had a strong association with myocardial perfusion, followed in importance by ACE D and ApoE g.-219T alleles [23].

There is also a correlation between arterial pressure control and lipid metabolism with the expression of ACE. This could be explained by the increased activity of ACE and the expected augmented expression of angiotensin II.

The conversion of angiotensin-1 (AT1) to angiotensin-2 (AT2) by ACE produces an increase in arterial blood pressure mediated via vasoconstriction, increased release of aldosterone that stimulates sympathetic nervous system, cell growth induction, etc. However, it also promotes cell migration, smooth muscle mitosis, and an increase in collagen synthesis, causing thickening of vascular walls and myocardium, promoting fibrosis. AT2 also has proinflammatory actions via induction of TNF $\alpha$ and IL- 6 production, both molecules promote the atherosclerotic plaque formation.

Certain polymorphisms of ACE are considered strong risk factors for the development of coronary artery disease and acute myocardial infarction, especially in low-risk populations. The insertion/deletion (I/D) ACE gene, in chromosome $17 \mathrm{q} 23$, provokes an increase of the expression levels, and in consequence, AT2 augmented expression. The increase in AT2 produces greater vasoconstriction, an increase in aldosterone synthesis, and sodium retention. It also favors a prothrombotic 


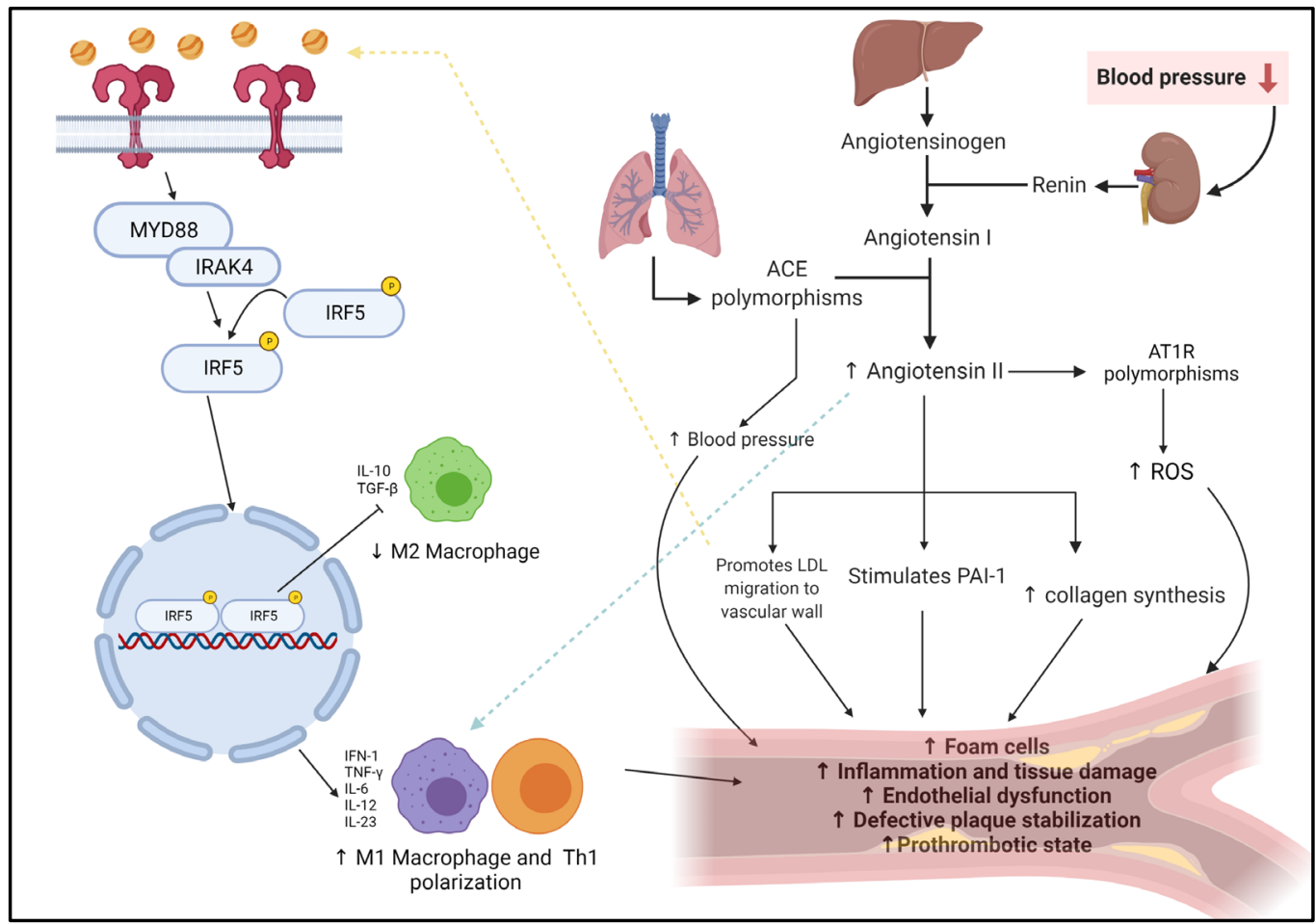

Figure 3. Relationship between interferon regulatory factor 5 and renin-angiotensin-aldosterone system in promoting atherosclerosis and cardiovascular disease

sate by stimulating plasminogen activator inhibitor 1 (PAI-1) and platelet adhesion.

The atherosclerotic process can be divided in 4 principal events: 1) endothelial dysfunction; 2) lipid and cholesterol deposits; 3) inflammatory reactions; 4) fibrous plaque formation with smooth muscle migration. This way, the principal trigger in the formation of atherosclerotic plaque is the LDL oxidation, where AT2 exerts a proatherogenic effect. This is due to the induction of native oxidized LDL aggregation on the vascular wall [24].

\section{Conclusion}

Recent studies have demonstrated the high influence that exerts IRF5 and RAAS polymorphisms on pathological and physiological states. They are implicated not only in cardiac diseases as well as in autoimmune and inflammatory diseases. IRF5 expression is directly related to atherosclerotic disease (Figure 3), it promotes among other effects TNF $\alpha$, IFN $\gamma$, and IL- 6 expression. Certain RAAS polymorphisms induce a proinflammatory state that ultimately leads to coronary artery disease and other related entities. A better understanding of the genetic basis of coronary artery disease may result in novel therapies and improved patient outcomes.

\section{Acknowledgements}

Special thanks to Dr. Nilda Espinola-Zavaleta and AFINES program. 


\section{Conflicts of Interest}

The authors declare no conflicts of interest regarding the publication of this paper.

\section{References}

[1] Narro, J. (2018) Enfermedades No Transmisibles Situación y Propuestas de Acción: Una Perspectiva desde la Experiencia de México. Secretaría de Salud, México.

[2] Chávez, R., Ramírez, J.A. and Casanova, J.M. (2003) La cardiopatía coronaria en México y su importancia clínica, epidemiológica y preventiva. Archivos de Cardiología de México, 73, 105-114. https://www.medigraphic.com/cgi-bin/new/resumen.cgi?IDARTICULO=70

[3] Almuttaqi, H. and Udalova, I. (2018) Advances and Challenges in Targeting IRF5, a Key Regulator of Inflammation. The Febs Journal, 286, 1624-1637. https://doi.org/10.1111/febs.14654

[4] Angelidis, G., Samara, M., Papathannasiou, M., Satra, M., Valotassoiu, V., Tsougos, I., et al. (2017) Impact of Renin-Angiotensin-Aldosterone System Polymorphisms on Myocardial Perfusion: Correlations with Myocardial Single Photon Emission Omputes Thomography-Derived Parameters. Journal of Nuclear Cardiology, 26, 1298 1308. https://doi.org/10.1007/s12350-017-1181-8

[5] Abbas, K., Lichtman, A. and Pillai, S. (2018) Cellular and Molecular Immunology. 9th Edition, Elsevier, Amsterdam, 4, 57-93.

[6] Krausgruber, T., Blazex, K., Smallie, T., Alzabin, S., Lockstone, H., Sahgal, N., Hussell, T., Feldman, M. and Udalova, I. (2011) IRF5 Promotes Inflammatory Macrophage Polarization and TH1-TH17 Responses. Nature Immunology, 12, 231-238. https://doi.org/10.1038/ni.1990

[7] Khoyratty, T.E. and Udalova, I.A. (2018) Diverse Mechanisms of IRF5 Action in Inflammatory Responses. International Journal of Biochemistry \& Cell Biology, 99, 3842. https://doi.org/10.1016/j.biocel.2018.03.012

[8] Thompson, C.D., Matta, B. and Barnes, B.J. (2018) Therapeutic Targeting of IRFs: Pathway-Dependence or Structure-Based? Frontiers in Immunology, 9, 2622. https://doi.org/10.3389/fimmu.2018.02622

[9] Weiss, M., Byrne, A.J., Blazek, K., Saliba, D.G., Pease, J.E., Perocheau, D., Feldmann, M. and Udalova, I.A. (2015) IRF5 Controls Both Acute and Chronic Inflammation. Proceedings of the National Academy of Sciences of the United States, 112, 1100111006. https://doi.org/10.1073/pnas.1506254112

[10] Watkins, A., Yasuda, K., Wilson, G., Aprahamian, T., Xie, Y., Maganto-Garcia, E., et al. (2015) IRF5 Deficiency Ameliorates Lupus but Promotes Atherosclerosis and Metabolic Dysfunction in a Mouse Model of Lupus-Associated Atherosclerosis. The Journal of Immunology, 194, 1467-1479. https://doi.org/10.4049/jimmunol.1402807

[11] Eames, H., Corbin, A. and Udalova, I. (2016) Interferon Regulatory Factor 5 in Human Autoimmunity and Murine Models of Autoimmune Disease. Translational Research, 167, 167-182. https://doi.org/10.1016/j.trsl.2015.06.018

[12] Lee, M.N., Ye, C., Villani, A.C., Raj, T., Li, W., Eisenhaure, T.M., Imboywa, T.H., Chipendo, P.I., et al. (2014) Common Genetic Variants Modulate Pathogen Sensing Responses in Human Dendritic Cells. Science, 343, 1119-1130. https://doi.org/10.1126/science.1246980

[13] Kim, S., Becker, J., Bechheim, M., Kaiser, V., Noursadeghi, M., Fricker, N., et al. (2014) Characterizing the Genetic Basis of Innate Immune Response in TLR4-Ac- 
tivated Human Monocytes. Nature Communications, 5, Article No. 5236. https://doi.org/10.1038/ncomms6236

[14] Sindhu, S., Thomas, R., Kochumon, S., Wilson, A., Abu-Farha, M., Bennakhi, A. Al-Mulla, F. and Ahmad, R. (2019) Increased Adipose Tissue Expression of Interferon Regulatory Factor (IRF)-5 in Obesity: Association with Metabolic Inflammation. Cells, 8, 1418. https://doi.org/10.3390/cells8111418

[15] Dalmas, E., Toubal, A., Alzaid, F., Blazek, K., Eames, H., Lebozec, K., et al. (2015) IRF5 Deficiency in Macrophages Promotes Beneficial Adipose Tissue Expansion and Insulin Sensitivity during Obesity. Nature Medicine, 21, 610-618.

https://doi.org/10.1038/nm.3829

[16] Byrne, A., Weiss, M., Mathie, S., Walker, S., Eames, H., Saliba, D., et al. (2016) A Critical Role for IRF5 in Regulating Allergic Airway Inflammation. Mucosal Immunology, 10, 716-726. https://doi.org/10.1038/mi.2016.92

[17] Mälarstig, A., Sigurdsson, S., Eriksson, P., Paulsson-Berne, G., Hedin, U., Wallentin, L., Siegbahn, A., Hamsten, A. and Syvänen, A.C. (2008) Variants of the Interferon Regulatory Factor 5 Gene Regulate Expression of IRF5 mRNA in Atherosclerotic Tissue But Are Not Associated with Myocardial Infarction. American Heart Association Journals, 28, 975-982. https://doi.org/10.1161/ATVBAHA.108.163733

[18] Fioranelli, M., Bottaccioli, A.G., Bottaccioli, F., Bianchi, M., Rovesti, M. and Roccia, M.G. (2008) Stress and Inflammation in Coronary Artery Disease: A Review Psychoneuroendocrine Immunology-Based. Frontiers in Immunology, 9, 2031. https://doi.org/10.3389/fimmu.2018.02031

[19] Numaguchi, Y., Ishii, M., Kubota, R., Morita, Y., Yamamoto, K., Matsushita, T., et al. (2009) Ablation of Angiotensin IV Receptor Attenuates Hypofibrinolysis via PAI-1 Downregulation and Reduces Occlusive Arterial Thrombosis. Arteriosclerosis, Thrombosis, And Vascular Biology, 29, 2102-2108. https://doi.org/10.1161/ATVBAHA.109.195057

[20] Zhu, M., Lin, J., Wang, C., Yang, M., Lv, H., Yang, M. and Jiang, J. (2019) The Relationship among Angiotensinogen Genes Polymorphisms and hs-CRP and Coronary Artery Disease. Journal of Clinical Laboratory Analysis, 33, e22881. https://doi.org/10.1002/jcla.22881

[21] Bonfim-Silva, R., Guimaraes, L.O., Souza, J., Pereira, J.F., Leal, A.A. and Souza D.L. (2016) Case-Control Association Study of Polymorphisms in the Angiotensinogen and Angiotensin-Converting Enzyme Genes and Coronary Artery Disease and Systemic Artery Hypertension in African-Brazilians and Caucasian-Brazilians. Journal of Genetics, 95, 63-69. https://doi.org/10.1007/s12041-015-0599-5

[22] Abd El-Aziz, T.A., Hussein, Y.M., Mohamed, R.H. and Shalaby, S.M. (2012) ReninAngiotensin System Genes Polymorphism in Egyptians with Premature Coronary Artery Disease. Gene, 498, 270-275. https://doi.org/10.1016/j.gene.2012.02.033

[23] Georgoulias, P., Wozniak, G., Samara, M., Chiotoglou, I., Kontos, A., Tzavara, C., Valotassiou, V., Georgitsi, M., Aleporou-Marinou, V., Patrinos, G.P. and Kollia, P. (2009) Impact of ACE and ApoE Polymorphisms on Myocardial Perfusion: Correlation with Myocardial Single Photon Emission Computed Tomographic Imaging. Journal of Human Genetics, 54, 595-602. https://doi.org/10.1038/jhg.2009.83

[24] Riad, M., Adhikari, P., Bhattarai, S., et al. (2021) Risk Assessment Using the Association Between Renin-Angiotensin Genes Polymorphisms and Coronary Artery Disease. Cureus, 13, e14083. https://doi.org/10.7759/cureus.14083 